\title{
Chapter 14 \\ Is there a Role for Adjuvant Targeted and Immunotherapies in Patients with Locoregionally-Advanced Head and Neck Cancer?
}

\author{
Kevin J. Harrington
}

\section{Introduction}

Although surgery and radiotherapy are the main curative treatment modalities in patients with locoregionally-advanced squamous cell cancers of the head and neck (LA-SCCHN), their use as single modality therapies or combined as dual modality (surgery and adjuvant post-operative radiotherapy) treatment is associated with unacceptably poor outcomes for many patients. Consequently, the development of curative therapies for LA-SCCHN has seen an inexorable shift towards combinatorial approaches that include systemic treatments delivered alongside surgery and/or radiotherapy.

As is frequently the case in oncology, clinicians have used the lessons learned in treating patients in the context of relapsed and/or metastatic head and neck cancer to provide useful indicators towards therapeutic approaches that can be employed effectively in the locoregionally-advanced setting. Building on data demonstrating the benefit of systemic platin-based chemotherapy $[1,2]$ with or without epidermal growth factor receptor (EGFR) inhibition [3, 4] in patients with relapsed and/or metastatic head and neck cancer, clinicians have established a robust body of evidence to support the use of systemic agents in the context of treating LA-SCCHN. Thus, multimodality regimens in which radiotherapy is delivered with the addition of concomitant platin-based chemotherapy $[5,6]$ or a monoclonal antibody that targets EGFR [7, 8] have become standards-of-care in younger patients of good performance status. However, similar approaches involving the use of either chemotherapy or EGFR inhibition in the context of adjuvant therapy delivered after definitive or post-operative (chemo)radiotherapy have not, as yet, resulted in practice-changing outcomes.

K. J. Harrington $(\varangle)$

Head of Division of Radiotherapy and Imaging, The Institute of Cancer Research,

London, UK

e-mail: Kevin.Harrington@icr.ac.uk 
In the case of immune checkpoint inhibitors (ICPI), there have also been attempts to extend the principle of applying that which is effective in the relapsed and/or metastatic setting to earlier time points within the patient pathway. ICPIs have been shown to be more effective than standard-of-care chemotherapy in both first- [9] and second-line [10-13] treatment of relapsed and/or metastatic head and neck cancer and have become new global gold-standard therapies. Importantly, in each of the practice-changing trials of ICPIs in head and neck cancer, patients have received prolonged dosing with immunotherapy and many have achieved durable and possibly permanent remissions. In many ways, the use of prolonged dosing regimens with ICPIs, even in patients who have achieved radiological complete remissions, can be seen as mimicking a course of adjuvant therapy. It is, therefore, no surprise that a number of clinical trials are currently addressing the question of using ICPIs in the adjuvant setting after the delivery of curative-intent treatment regimens, including studies involving adjuvant therapy after definitive chemoradiotherapy or surgery followed by post-operative chemoradiotherapy.

Therefore, in this chapter, we will discuss the potential role of adjuvant therapies in patients with LA-SCCHN. Data on the use of cytotoxic chemotherapy agents will be reviewed first in order to provide a context for more recent attempts to develop effective adjuvant approaches based on EGFR-targeted therapies and ICPIs.

\section{Adjuvant Post-Operative Chemoradiotherapy Improves Outcomes in LA-SCCHN}

Post-operative radiotherapy (PORT) is considered when the risk of recurrence of disease above the clavicles exceeds $20 \%$ (either at the mucosal site or in surgicallytreated and -untreated nodal levels). Absolute indications for PORT include close (less than $5 \mathrm{~mm}$ ) or involved (positive) margins at the primary tumour resection site, two or more involved cervical lymph nodes, extracapsular spread and invasion of the soft tissues of the neck. The presence of lymphovascular space invasion and perineurial invasion are relative indications for PORT that are considered in association with other factors [14].

The role of adjuvant post-operative chemoradiotherapy (POCRT), as opposed to PORT, was clarified in two seminal studies published in $2004[15,16]$ and summarised in Fig. 14.1. It is important to emphasise that, in both of these trials, the delivery of chemotherapy was restricted solely to the period of time during which the patient received adjuvant post-operative radiotherapy. In other words, there was no use of chemotherapy in a fashion that might be considered as true adjuvant therapy. The inclusion criteria for the RTOG 9501 [15] and EORTC 22931 [16] studies were slightly different, but the overall findings were remarkably similar. In the RTOG study, loco-regional control and disease-free survival were increased by the addition of concomitant cisplatin to PORT. There was a $10 \%$ improvement in 2-year loco-regional control (82\% vs $72 \%$ ) in favour of POCRT. In the EORTC study, 

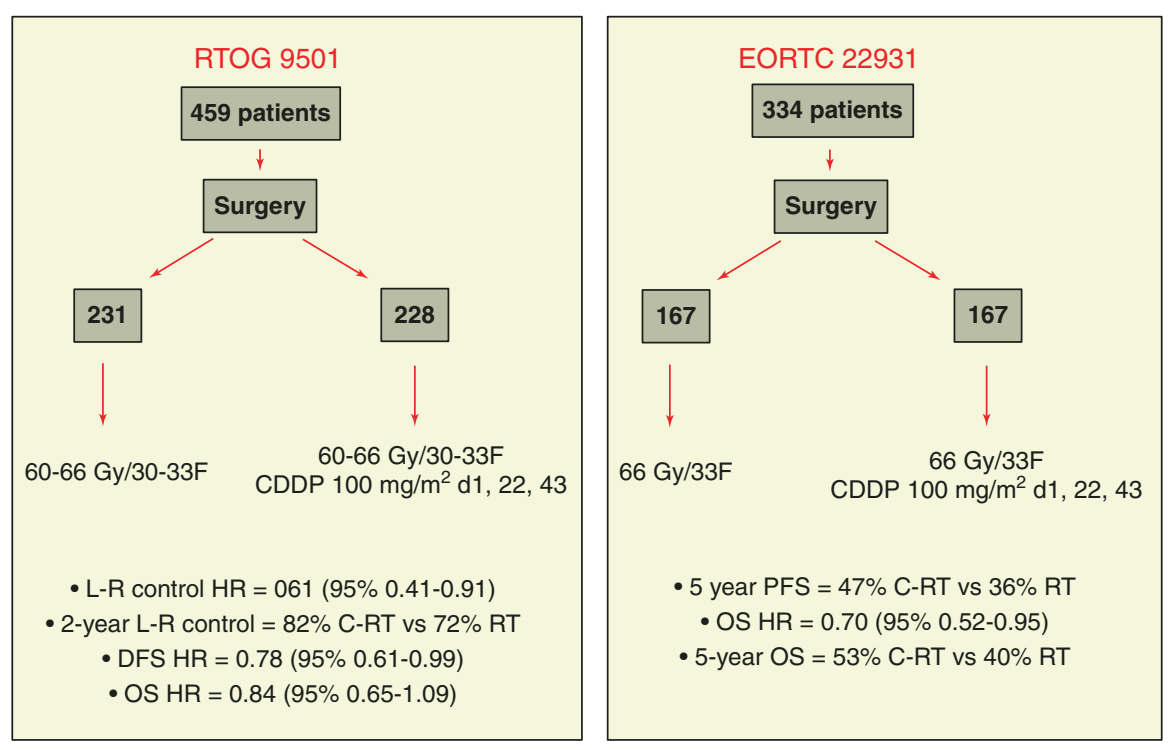

Fig. 14.1 Trial designs and main findings of the RTOG 9501 and EORTC 22931 trials of postoperative radiotherapy versus post-operative chemoradiotherapy

5-year progression-free and overall survival rates were increased by the addition of concomitant cisplatin to PORT.

The authors of those two studies subsequently amalgamated the datasets to derive consensus indications for POCRT [17]. They found that extracapsular spread in lymph node disease and the presence of microscopically involved resection margins were the only risk factors for which the impact of POCRT was significant in both trials. Therefore, these two factors have been widely adopted as absolute indications for POCRT across the globe. The combined analysis also suggested a trend in favour of POCRT in patients with stage III/IV disease, those with perineurial invasion, lymphovascular space emboli or level IV and V lymph nodes in the case of oral cavity or oropharyngeal cancers. These factors have not been accepted widely as absolute indications for POCRT.

\section{Adjuvant Chemotherapy Is Not Effective in LA-SCCHN}

The Meta-Analysis of Chemotherapy on Head and Neck Cancer (MACH-NC) collaborative group was originally published in 2000 and represented a landmark study that fundamentally changed the standard-of-care treatment paradigms for patients with stage III/IV disease [5]. That meta-analysis addressed three specific issues: (i) the effect on survival outcomes of adding chemotherapy to locoregional treatment; (ii) the effect of different chemotherapy schedules; and (iii) the role of induction/ 
neoadjuvant chemotherapy in laryngeal preservation. Data relating to the first two questions have provided clear guidance on the absence of a defined role for adjuvant cytotoxic chemotherapy in LA-SCCHN. Thus, although the meta-analysis, which included 63 trials involving 10,741 patients, demonstrated that survival following definitive local therapy was significantly improved with the addition of chemotherapy, neither induction nor adjuvant chemotherapy was seen to have had a significant effect on survival outcomes.

In view of the heterogeneity of the studies included in the original MACH-NC meta-analysis, a further study was conducted in which the group updated their results by including individual patient data from randomised trials that were conducted between 1994 and 2000 [6]. Data from a total of 87 randomised trials were available for the analyses. Because some trial arms were used more than once in the analysis, the total number of comparisons in the updated meta-analysis was 108 comprising data on 17,493 patients. The majority of new trial data were specifically related to the use of concomitant chemotherapy and resulted in the meta-analysis being able to draw much clearer conclusions about the value of this treatment approach. As with the previous analysis, there was a significant effect of the timing of chemotherapy with concomitant chemotherapy clearly offering the greatest benefit (hazard ratio 0.81 (95\% CI 0.78-0.86). Once again, neither induction (hazard ratio 0.96 (95\% CI 0.90-1.02) nor adjuvant (hazard ratio 1.06 (95\% CI 0.95-1.18) chemotherapy was found to be better than locoregional therapy alone. As a consequence, there is broad agreement across the globe that adjuvant chemotherapy has no established role when delivered after definitive surgery, radiotherapy, or radical/ post-operative chemoradiotherapy.

\section{Epidermal Growth Factor Receptor-Targeted Therapies in LA-SCCHN}

SCCHN very frequently show upregulated EGFR signalling. EGFR is a member of the c-erbB/HER family of transmembrane type I receptor tyrosine kinases, which comprises a total of four members (EGFR/c-erbB-1/HER1, c-erbB-2/neu/HER-2, c-erbB-3/HER-3, c-erbB-4/HER-4 [18, 19]). HER-family receptor proteins share a common structure, consisting of a glycosylated extracellular ligand-binding domain, a hydrophobic trans-membrane component and an intracellular domain with tyrosine kinase activity. When the specific (cognate) ligand binds to its ligand-binding domain on the extracellular component of a HER-family member, it causes receptor dimerization and activation of the kinase domain. This, in turn, mediates phosphorylation of target proteins, which triggers a cascade of intracellular secondary messengers that alter patterns of gene expression. In this way, binding of a protein on the cell surface can influence the cell's behaviour. It is important to note that there is no ligand for the c-erbB2/HER2 receptor and that the c-erbB3/HER3 receptor has no kinase activity. Nonetheless, these receptors are able to participate in signalling 
by dimerizing with appropriate partners. For example, EGFR:c-erbB3 (HER1/ HER3) and c-erbB-2:c-erbB-3 (HER2/HER3) heterodimers can exploit ligand binding to the HER3 component and kinase-mediated signalling from the EGFR or HER2 component of the partnership.

Normally, activation of HER-family receptors by their cognate ligands (growth factors) is very tightly controlled-through regulation of the expression of the receptor, the availability of the ligand and the ability of the cell to de-phosphorylate activated receptors. In this way, proliferation of normal tissues is carefully regulated to avoid unnecessary or unscheduled cell growth. In contrast, in SCCHN there is very frequently a state of independence from normal regulatory mechanisms that is driven through a number of different processes. These include: (i) manufacture and release of growth factors that stimulate HER-family receptors on the malignant cell (autocrine signalling) and on neighbouring malignant (and normal) cells (paracrine signalling); (ii) altering the number, structure or function of the surface growth factor receptors expressed on tumour cells; and (iii) by altering the signalling pathways downstream of the receptor. In contrast to other tumour types, in which EGFR gene amplification or mutation is common (e.g. lung adenocarcinoma), overexpression of the receptor, without gene amplification, is the dominant process whereby EGFR affects the pathobiology of SCCHN. The roles of HER2, HER3 and HER4 in SCCHN remain unclear. However, it is known that HER2/HER3 heterodimers are potent inducers of the PI3-kinase anti-apoptotic pathway [20] and this may be relevant to particular subsets of SCCHN, including human papillomavirus (HPV)related disease $\mathrm{s}^{37}$.

Irrespective of the fact that SCCHN rarely shows evidence of EGFR mutation, the evidence of single-agent responses to HER-family-targeted therapies strongly supports the notion that these tumours can be reliant on signalling through these pathways in order to maintain the malignant phenotype. This reliance on activation of an oncogenic driver has been called "oncogene addiction" [21] and is seen as a potential point of therapeutic attack against a range of tumour types. Therefore, HER-family receptors represent attractive therapeutic targets in SCCHN and two main classes of drugs, monoclonal antibodies (MAB) and small molecule tyrosine kinase inhibitors (smTKI), have been developed. MAB are large molecules directed against the extracellular domain of the receptor, while smTKI inhibit the intracellular kinase domain of the receptor.

\section{Anti-EGFR Monoclonal Antibodies Are Not Used as Adjuvant Therapies for LA-SCCHN}

Anti-EGFR-targeted monoclonal antibodies (cetuximab, zalutumumab, panitumumab, nimotuzumab) have been extensively tested in patients with relapsed and/ or metastatic head and neck cancers. Cetuximab has been shown to improve the outcome of first-line palliative chemotherapy [3]. In the EXTREME study, 442 
eligible patients with untreated recurrent and/or metastatic SCCHN received cisplatin or carboplatin plus 5-fluorouracil every 3 weeks for a maximum of 6 cycles. Cetuximab (400 mg/m ${ }^{2}$ loading dose, then $250 \mathrm{mg} / \mathrm{m}^{2}$ per week) was administered to 222 randomly selected patients. Patients in the cetuximab arm who showed stable disease or treatment response were planned to continue with maintenance cetuximab until disease progression or unacceptable toxicity. This study showed that the cetuximab/platinum/5-fluorouracil combination prolonged median overall survival from 7.4 months to 10.1 months $(\mathrm{P}=0.04)$. There were also increases in the median progression-free survival time (3.3 to 5.6 months; $\mathrm{P}<0.001$ ) and the response rate ( $20 \%$ to $36 \%$; $\mathrm{P}<0.001$ ) [3]. Consequently, the EXTREME regimen was adopted as a gold-standard treatment for relapsed/metastatic head and neck cancer. In contrast, neither panitumumab [22] nor zalutumumab [23] has been registered for the treatment of relapsed and/or metastatic head and neck cancer following negative phase III trials, although the data from those trials was strongly suggestive of activity of those agents. The use of nimotuzumab is largely restricted to India and there are very limited data relating to its use in recurrent and/or metastatic SCCHN [24].

Cetuximab has also been approved as part of a curative regimen for LA-SCCHN. In a phase III study of 424 subjects with locally or regionally advanced SCCHN, locoregional control (median 24.4 vs. 14.9 months; hazard ratio: $0.68 ; \mathrm{P}=0.005$ ) and overall survival (median 49.0 vs. 29.3 months; hazard ratio: $0.74 ; \mathrm{P}=0.03$ ) were significantly prolonged in patients receiving radiotherapy and cetuximab compared to those treated with radiotherapy alone [7, 8]. However, in this study, there was no continued, adjuvant use of cetuximab beyond the completion of radiotherapy. In addition, both zalutumumab and panitumumab have been tested in combination with radiation/chemoradiation, again without any attempt to use them in an adjuvant phase beyond the completion of definitive treatment. Neither of these agents improved outcomes when compared to the standard therapy arms and they have not been approved in the context of LA-SCCHN [25-27]. Nimotuzumab is widely used with chemoradiotherapy in India, following the publication of a positive randomised phase III trial. However, once again, there was no use of nimotuzumab following completion of definitive loco-regional therapy [28] and, therefore, there is no evidential basis on which to deliver this therapy in an adjuvant setting.

\section{Small Tyrosine Kinase Inhibitors Are Not Effective Adjuvant Therapies in LA-SCCHN}

A number of agents have been developed to target HER-family members across a variety of different tumour types. In the context of head and neck cancer, gefitinib and erlotinib (EGFR/HER1 inhibitors) [29-31], lapatinib (HER1/HER2 inhibitor) [32, 33] and afatinib (pan-HER inhibitor) [34] have been most extensively investigated. Studies have included assessments of agents in the palliative setting for relapsed and/or metastatic disease. Despite the fact that these agents demonstrate 
single-agent activity in phase I/II trials, randomised studies have failed to demonstrate clinically meaningful survival advantage relative to standard-of-care treatment and none of them is in routine clinical use for patients with relapsed and/or metastatic disease. Specifically, a randomised phase III study was conducted to compare survival in 486 patients with recurrent or metastatic SCCHN treated with gefitinib 250 or $500 \mathrm{mg} /$ day or standard-of-care single-agent weekly methotrexate [35]. Neither of the gefitinib doses improved overall survival compared with methotrexate (hazard ratios 1.22 (95\% CI $0.95-1.57$ and 1.12 (95\% CI, 0.87-1.43), respectively. The median overall survivals were 5.6, 6.0, and 6.7 months for gefitinib $250 \mathrm{mg} /$ day, gefitinib $500 \mathrm{mg} / \mathrm{day}$, and intravenous methotrexate, respectively. Afatinib has been assessed in the phase III LUX head and neck-1 study in patients receiving second-line therapy for relapsed/metastatic SCCHN [36]. A total of 583 patients were treated with afatinib (322 patients) or methotrexate ((161 patients). Afatinib significantly increased median progression-free survival (2.6 versus 1.7 months, $\mathrm{p}=0.03$ ) but did not improve median overall survival (6.8 versus 6.0 months) relative to methotrexate. In an integrated analysis of quality of life, afatinib showed a delay in deterioration of global health status, pain and swallowing problems (all $\mathrm{p} \leq 0.03$ ) but such data were of insufficient weight to lead to regulatory approval of this therapy.

In the context of adjuvant maintenance therapy using HER-family-targeted therapies, there have been significant attempts to develop lapatinib and afatinib. A randomised phase III study of lapatinib administered concomitantly with chemoradiotherapy and as maintenance monotherapy in patients with high-risk surgically-treated SCCHN has been reported [37, 38]. Patients with resected stage II-IVA SCCHN, with a surgical margin $\leq 5 \mathrm{~mm}$ and/or extracapsular extension in metastatic cervical nodal disease were randomized to chemoradiotherapy (66 Gy total dose and $100 \mathrm{mg} / \mathrm{m}^{2}$ cisplatin administered on days 1, 22 and 43) plus placebo or lapatinib (1500 mg/day) prior to and during chemoradiotherapy, followed by 12 months of maintenance monotherapy (either placebo or lapatinib). Six hundred and eighty-eight patients were enrolled; 346 received lapatinib and 342 received placebo. With a median follow-up of 35.3 months, the study was terminated early due to the apparent plateauing of disease-free survival events. Median disease-free survivals were 53.6 months and "not reached" for lapatinib and placebo, respectively; hazard ratio 1.10 (95\% CI 0.85-1.43). No significant differences in diseasefree survival by HPV status or overall survival were observed between the two treatment arms. Similar numbers of patients in both treatment arms experienced adverse events, with more patients in the lapatinib arm experiencing serious events ( $48 \%$ vs $40 \%$ ). This study demonstrated that adding lapatinib to chemoradiotherapy and its use as long-term adjuvant therapy was safe, but did not offer any efficacy benefits compared with placebo in patients with surgically-treated high-risk SCCHN.

In the LUX head and neck-2 study, 617 patients were randomised to treatment (411 to afatinib and 206 to placebo) in a true adjuvant context [39, 40]. Eligible patients had histologically or cytologically confirmed LA HNSCC (Stage III, IVa or IVb SCC of the oral cavity, oropharynx or hypopharynx, or Stage IVa or IVb SCC of the larynx). Since HPV status was not determined for eligibility, unfavourable 
risk was defined as non-oropharynx primary site or oropharynx cancer in heavy smokers ( $>10$ pack years). Patients were required to have unresected disease prior to chemoradiotherapy. Concomitant definitive chemoradiotherapy had to have been completed no longer than 24 weeks prior to randomisation, comprising radiotherapy with curative intent to a minimum dose of 66 Gy in 33 fractions, and cisplatin or carboplatin. No evidence of disease was required on clinical and radiographic examinations (defined as no residual tumour after chemoradiotherapy (with or without $\mathrm{R} 0$ resection at the primary site or neck dissection). A pre-planned futility analysis, showed the study was unlikely to demonstrate a significant advantage with afatinib and the trial was halted early on the recommendation of the independent data-monitoring committee. Patients were discontinued from treatment and followup for disease recurrence and survival was stopped. The percentage of patients taking at least $80 \%$ of the planned study medication was lower for the afatinib group $(85.3 \%)$ than the placebo group $(98.5 \%)$, almost certainly reflecting the appreciable toxicity associated with chronic administration of this pan-HER-targeted oral medication. Median disease-free survival (DFS) by investigator review was 43.4 months (95\% CI 37.4-not estimable) with afatinib versus "not estimable" (95\% CI 40.1-not estimable) with placebo (HR 1.13, 95\% CI 0.81-1.57; stratified log-rank test $\mathrm{p}=0.48$ ). The DFS rate at 2 years was evaluated using the Kaplan-Meier method; the probability of being disease-free at 2 years was $67.2 \%$ in the afatinib group and $73.5 \%$ in the placebo group (estimated difference: $-6.3 \%$, 95\% CI -15.0-2.5; $\mathrm{p}=0.16)$. At the time of data cut-off for the futility analysis, overall survival data were immature. The effect of afatinib versus placebo on DFS was explored in preplanned subgroup analyses based on stratification factors, biomarker status, demographics, baseline disease characteristics and prior anti-cancer chemotherapy. These subgroup analyses were generally consistent with the primary analysis and showed no clear trend of benefit in any subgroup, although there was a slight benefit for afatinib patients with nodal status N2b-N3 (HR 0.82, 95\% CI 0.55-1.21).

\section{Immune Checkpoint Inhibitors as Adjuvant Therapies in LA-SCCHN}

In recent years, immunotherapy has emerged as a new pillar in the treatment of many solid cancers [41]. This renewed interest in immunotherapy has been underscored by huge advances in our understanding of the fundamental biological principles that guide the activity of the immune system. In particular, specific immune checkpoints have been discovered that are integral components of normal immune responses. In normal health, these checkpoints function as negative regulators or "brakes" on the normal immune response and prevent $\mathrm{T}$ cells from becoming chronically activated or aberrantly targeted against normal tissues. Many cancers exploit these inhibitory pathways in order to escape from immunosurveillance.

Proteins that are expressed on activated T cells, such as cytotoxic T-lymphocyteassociated protein-4 (CTLA-4) and programmed death-1 (PD-1), are key players 
that allow many cancers to evade anti-tumour immunity by interfering with the activation and effector phases of immune responses, respectively. In the context of relapsed and/or metastatic head and neck cancer, we have clear evidence that blockade of signalling through the PD-1 pathway (mediated by programmed death ligand-1 (PD-L1) expressed on the surface of cancer and other cells) can yield significant clinical responses. Indeed, we now have positive phase III trial data, initially in the second-line and, more recently, in the first-line setting, to show that anti-PD-1-targeted therapies are capable of significantly improving overall survival in patients with relapsed and/or metastatic head and neck cancers [9-13]. As with chemotherapy and HER-family-targeted therapies, such data have spurred on investigators to investigate the potential value of immune checkpoint inhibitors (ICPI) as adjuvant therapies for cancer.

A number of lines of evidence can be invoked to support the hypothesis that adjuvant ICPI therapy might be beneficial in patients treated for LA-SCCHN. First, there are data in patients with melanoma that demonstrate that the "baseline tumour size" is an independent, statistically significant predictor of overall survival in patients treated with anti-PD-1 ICPI [42]. In addition, phase III clinical trials have shown improvement in progression-free and overall survival endpoints for tumour types such as melanoma and lung cancer [43-46]. As a guide to the management of SCCHN, the data from the PACIFIC trial in lung cancer are most compelling because the patient population comprised those with locally-advanced, stage III non-small-cell lung cancer who had not progressed on chemoradiotherapy delivered with curative intent $[45,46]$. Interestingly, patients were required to commence adjuvant anti-PD-L1 therapy (durvalumab) within 42 days of completion of chemoradiotherapy.

At the time of writing, there are at least 3 major trials that have either completed recruitment or are still ongoing that address the question of adjuvant/maintenance ICPI in locally-advanced head and neck cancer. The designs of these trials are summarised in Figs. 14.2, 14.3 and 14.4.

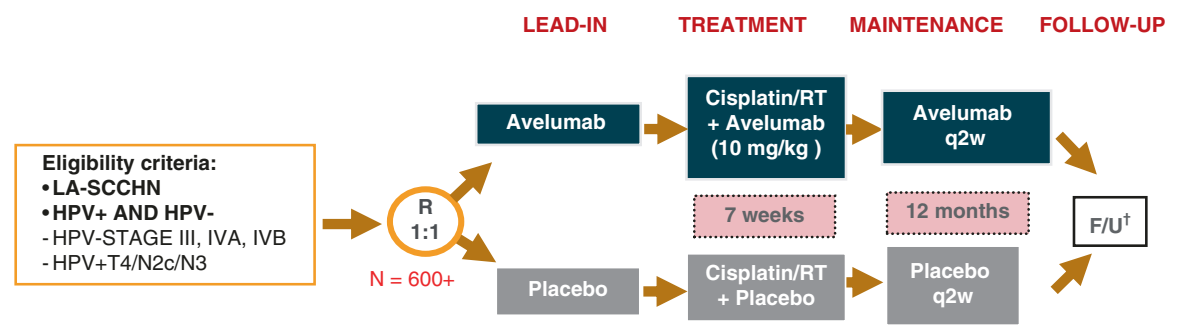

Key: F/U - follow-up; HPV - human papillomavirus; LA-SCCHN - locally-advanced squamous cell cancer of the head and neck; q2w - every 2 weeks; $\mathrm{R}$ - randomisation; RT - radiotherapy

Fig. 14.2 Javelin Head and Neck-100 study design. Key: $F / U$ follow-up; $H P V$ human papillomavirus; $L A-S C C H N$ locally-advanced squamous cell cancer of the head and neck; $q 2 w$ every 2 weeks; $R$ randomisation; $R T$ radiotherapy 
Local testing for $\mathrm{p} 16^{\star *}$

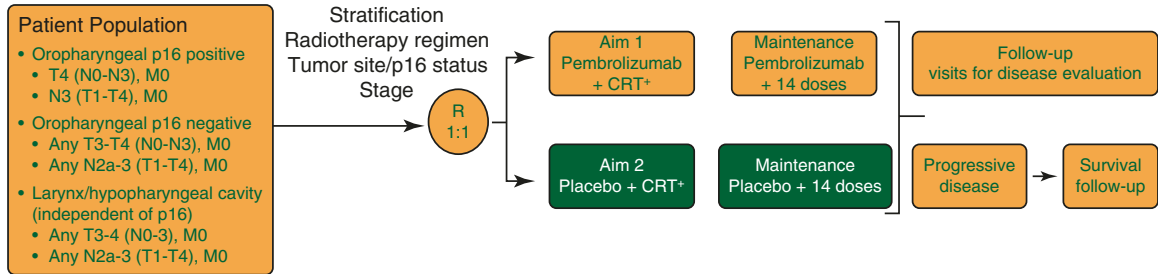

** Expression of P16 is highly correlated with HPV in oropharyngeal cancer. Tumors outside the oropharynx are considered HPV negative regardless of results of $\mathrm{p} 16$ staining.

+ Chemotherapy: Cisplatin 100 mg/m² x 3; RT: AFX - 200 cGY/6 per week, SFX - 200 cGy/5 per week

Fig. 14.3 KEYNOTE-412 clinical trial design. ${ }^{* *}$ Expression of P16 is highly correlated with HPV in oropharyngeal cancer. Tumors outside the oropharynx are considered HPV negative regardless of results of p16 staining. + Chemotherapy Cisplatin $100 \mathrm{mg} / \mathrm{m}^{2} \times 3 ; R T$ AFX $-200 \mathrm{cGY} / 6 \mathrm{per}$ week, SFX - 200 cGy/5 per week. Key: $A F X$ accelerated fractination; p16-positive/negative surrogate measure for human papillomavirus; $R$ randomisation; $R T$ radiotherapy; $S F X$ standard fractionation

\section{Stratification By:}

- Response to defeinitive local treatment (CR vs. PR or SD)

- HPV Status (positive vs. negative)

- $20 \%$ enrollment cap for HPV-positive

- Type of DLT (primary surgery vs. no primary surgery

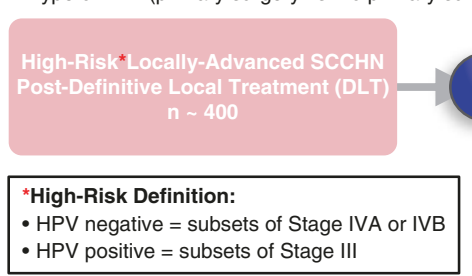

Atezolizumab

Q3W x 16 doses or 1 year, whichever is first

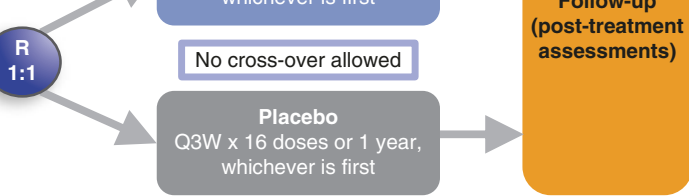

Key: CR - complete remission; DLT - definitive local treatment; HPV - human papillomavirus; PR - partial remission; Q3w - every 3 weeks; $\mathrm{R}$ - randomisation; RT - radiotherapy ; SD - stable disease

Fig. 14.4 IMvoke010 clinical trial design. Key: $C R$ complete remission; $D L T$ definitive local treatment; $H P V$ human papillomavirus; $P R$ partial remission; $Q 3 w$ every 3 weeks; $R$ randomisation; $R T$ radiotherapy; $S D$ stable disease

The Javelin Head and Neck 100 study was a randomized, double-blind, placebocontrolled, parallel-arm, superiority study of the anti-PD-L1 agent, avelumab, versus placebo. Patients with LA-SCCHN (oral cavity, oropharynx, larynx, or hypopharynx) who were eligible for definitive chemoradiotherapy were enrolled (details of patient groups are provided in Fig. 14.2). Patients were randomized to receive either avelumab or placebo plus standard-of-care chemoradiotherapy. Randomization was stratified by tumour stage ( $<\mathrm{T} 4$ vs T4), nodal stage (NO/ $\mathrm{N} 1 / 2 \mathrm{aN} 2 \mathrm{~b}$ vs N2c/N3), and HPV status (positive vs negative). There were three treatment phases in the study: lead-in phase; treatment phase; and maintenance phase. The primary endpoint of Javelin Head and Neck 100 is the progression-free survival (PFS) per modified Response Evaluation Criteria in Solid Tumours 
(RECIST) version v1.1 by investigator assessment. Secondary endpoints include overall survival, pathologic complete response, neck dissection, locoregional failure, objective response, distant metastatic failure, and duration of response, per modified RECIST v1.1 by investigator assessment. In March 2020, the study sponsors accepted the recommendation of the independent Data Monitoring Committee to terminate the JAVELIN Head and Neck 100 trial, as the study is unlikely to show a statistically significant improvement in the primary endpoint of PFS based on a pre-planned interim analysis [47]. A detailed analysis of the study findings are likely to be available for examination by the scientific community in 2021 .

KEYNOTE-412 (ClinicalTrials.gov, NCT03040999) has a similar design to Javelin Head and Neck 100 (Fig. 14.3). It is a randomized, double-blind, placebocontrolled, phase III study of pembrolizumab $200 \mathrm{mg}$ or placebo every 3 weeks in combination with chemoradiotherapy and as maintenance/adjuvant therapy for a total of 17 doses over one year [48]. Study recruitment has now closed, but patients remain in follow-up as the data mature. Eligible patients were randomly assigned 1:1 to pembrolizumab or placebo with stratification according to radiotherapy regimen (accelerated versus standard fractionation), tumor site, p16 status (orophar$\mathrm{ynx}-\mathrm{p} 16$ positive vs oropharynx $-\mathrm{p} 16$ negative or larynx/hypopharynx/oral cavity cancer), and tumour stage (III vs IV, AJCC TNM Version 7). Treatment is continued until centrally confirmed disease progression, unacceptable toxicity, intercurrent illness that prevents further administration of treatment, investigator's decision to withdraw the patient, non-adherence to treatment or trial procedures, administrative reasons requiring cessation of treatment, or the patient has received 17 administrations of pembrolizumab/placebo (approximately 1 year). The trial is split into three treatment phases. The first phase includes the pembrolizumab/placebo priming dose, followed by chemoradiotherapy in combination with two additional pembrolizumab/placebo doses given every 3 weeks (duration, 8 weeks). The second phase includes pembrolizumab/placebo maintenance/adjuvant dosing (14 doses over about a year) during post-treatment follow-up. The third phase includes posttreatment follow-up. The primary end point of the trial is event-free survival (EFS) using RECIST v1.1. EFS is defined as the time from the date of randomization to the date of first record of (1) progression per RECIST v1.1 by blinded independent central review ([a] locoregional progression or recurrence or [b] distant metastasis), (2) salvage surgery at the primary tumour site when invasive cancer is present, (3) neck dissection performed $>20$ weeks after completion of CRT when invasive cancer is present, or (4) death from any cause. The key secondary end point is overall survival, which is defined as the time from randomization to death from any cause. Other secondary end points include safety and patient-reported outcomes (PROs); PROs are assessed using the European Organisation for the Research and Treatment of Cancer Quality of Life Questionnaire (EORTC QLQ) core 30 items (C30) and head and neck module (H\&N35) as well as the EuroQoL-5D (EQ-5D). Exploratory end points include potential predictive biomarkers and immune dynamics in the subgroup of patients with oropharyngeal p16-negative or larynx/hypopharynx/oral cavity HNSCC and the overall population. 
The ImVoke-10 study (ClinicalTrials.gov NCT03452137) is a phase III, multicentre, randomized, double-blind, placebo-controlled trial of the anti-PD-L1 ICPI, atezolizumab, as adjuvant therapy following definitive local therapy for high-risk LA-SCCHN [49]. Its design is depicted in Fig. 14.4. Approximately 400 patients will be randomised between atezolizumab and placebo given in a truly adjuvant setting (Fig. 14.4). The co-primary endpoints are independent review facility assessed EFS (per RECIST v1.1) or death from any cause and overall survival.

\section{Summary and Conclusions}

Despite relatively poor outcomes for many patients who present with LA-SCCHN, we have, thus far, failed to develop effective adjuvant therapies to prevent locoregional and/or metastatic relapse following definitive local therapy. Extensive effort has been exerted in attempting to develop adjuvant chemotherapy schedules, but to no avail. Similarly, attempts to exploit the concept of "oncogene addiction" by using adjuvant HER-family-targeted therapies have not been successful. There is no evidence that either small molecule inhibitors or monoclonal antibodies given in the adjuvant situation can favourably alter recurrence rates or survival outcomes. Currently, most effort is being channeled into studies that seek to evaluate the potential role of ICPI as adjuvant therapies. Considerations around these trial designs are complex, since both the Javelin Head and Neck 100 and the KEYNOTE-412 studies involve a combination of concomitant (with chemoradiotherapy) and adjuvant ICPI therapy. Neither study is designed to allow separate evaluation of the role of the concomitant versus the adjuvant components of the therapeutic package. Nevertheless, given the remarkable results in the context of relapsed and/or metastatic head and neck cancer, there is cause for optimism that we may be able to improve outcomes for our patients.

\section{References}

1. Forastiere AA, Metch B, Schuller DE, et al. Randomized comparison of cisplatin plus fluorouracil and carboplatin plus fluorouracil versus methotrexate in advanced squamous-cell carcinoma of the head and neck: a southwest oncology group study. J Clin Oncol. 1992;10:1245-51.

2. Forastiere AA. Chemotherapy of head and neck cancer. Ann Oncol. 1992;3(Suppl 3):11-4.

3. Vermorken JB, Mesia R, Rivera F, et al. Platinum-based chemotherapy plus cetuximab in head and neck cancer. N Engl J Med. 2008;359:1116-27.

4. Burtness B, Goldwasser MA, Flood W, et al. Phase III randomized trial of cisplatin plus placebo compared with cisplatin plus cetuximab in metastatic/recurrent head and neck cancer: an eastern cooperative oncology group study. J Clin Oncol. 2005;23:8646-54.

5. Pignon J, Bourhis J, Domenge C, Designé L. Chemotherapy added to locoregional treatment for head and neck squamous-cell carcinoma: three meta-analyses of updated individual data. Lancet. 2000;355:949-55. 
6. Pignon JP, le Maître A, Maillard E, Bourhis J. MACH-NC collaborative group. Meta-analysis of chemotherapy in head and neck cancer (MACH-NC): an update on 93 randomised trials and 17,346 patients. Radiother Oncol. 2009;92:4-14.

7. Bonner JA, Harari PM, Giralt J, et al. Radiotherapy plus cetuximab for squamous-cell carcinoma of the head and neck. N Engl J Med. 2006;354:567-78.

8. Bonner JA, Harari PM, Giralt J, et al. Radiotherapy plus cetuximab for locoregionally advanced head and neck cancer: 5-year survival data from a phase 3 randomised trial, and relation between cetuximab-induced rash and survival. Lancet Oncol. 2010;11:21-8.

9. Burtness B, Harrington KJ, Greil R, et al. Pembrolizumab alone or with chemotherapy versus cetuximab with chemotherapy for recurrent or metastatic squamous cell carcinoma of the head and neck (KEYNOTE-048): a randomised, open-label, phase 3 study. Lancet. 2019;394(10212):1915-28.

10. Ferris RL, Blumenschein G, Fayette J, et al. Nivolumab for recurrent squamous-cell carcinoma of the head and neck. N Engl J Med. 2016;375:1856-67.

11. Gillison ML, Blumenschein G Jr, Fayette J, et al. CheckMate 141: 1-year update and subgroup analysis of Nivolumab as first-line therapy in patients with recurrent/metastatic head and neck cancer. Oncologist. 2018;23:1079-82.

12. Ferris RL, Blumenschein G Jr, Fayette J, et al. Nivolumab vs investigator's choice in recurrent or metastatic squamous cell carcinoma of the head and neck: 2-year long-term survival update of CheckMate 141 with analyses by tumor PD-L1 expression. Oral Oncol. 2018;81:45-51.

13. Cohen EEW, Soulieres D, Le Tourneau C, et al. Pembrolizumab versus methotrexate, docetaxel, or cetuximab for recurrent or metastatic head-and-neck squamous cell carcinoma (KEYNOTE-040): a randomised, open-label, phase 3 study. Lancet. 2019;393(10167):156-67.

14. NCCN Clinical Practice Guidelines in Oncology (NCCN Guidelines $\left.{ }^{\circledR}\right)$ : Head and Neck Cancers. Version 2. 2013. NCCN.org.

15. Cooper JS, Pajak TF, Forastiere AA, et al. Postoperative concurrent radiotherapy and chemotherapy for high-risk squamous-cell carcinoma of the head and neck. N Engl J Med. 2004;350:1937-44.

16. Bernier J, Domenge C, Ozsahin M, et al. Postoperative irradiation with or without concomitant chemotherapy for locally advanced head and neck cancer. N Engl J Med. 2004;350:1945-52.

17. Bernier J, Cooper JS, Pajak TF, et al. Defining risk levels in locally advanced head and neck cancers: a comparative analysis of concurrent postoperative radiation plus chemotherapy trials of the EORTC (\#22931) and RTOG (\# 9501). Head Neck. 2005;27:843-50.

18. Rogers SJ, Harrington KJ, Rhys Evans P, et al. Biological significance of c-erbB family oncogenes in head and neck cancer. Cancer Metastasis Rev. 2005;24:47-69.

19. Khademi B, Shirazi FM, Vasei M, et al. The expression of p53, c-erbB-1 and c-erbB-2 molecules and their correlation with prognostic markers in patients with head and neck tumors. Cancer Lett. 2002;184:223-30.

20. Hellyer NJ, Kim MS, Koland JG. Heregulin-dependent activation of phosphoinositide 3-kinase and Akt via the ErbB2/ErbB3 co-receptor. J Biol Chem. 2001;276:42153-61.

21. Weinstein B, Joe A. Oncogene addiction. Cancer Res. 2008;68:3077-80.

22. Vermorken JB, Stöhlmacher-Williams J, Davidenko I, et al. Cisplatin and fluorouracil with or without panitumumab in patients with recurrent or metastatic squamous-cell carcinoma of the head and neck (SPECTRUM): an open-label phase 3 randomised trial. Lancet Oncol. 2013; 14:697-710.

23. Machiels JP, Subramanian S, Ruzsa A, et al. Zalutumumab plus best supportive care versus best supportive care alone in patients with recurrent or metastatic squamous-cell carcinoma of the head and neck after failure of platinum-based chemotherapy: an open-label, randomised phase 3 trial. Lancet Oncol. 2011;12:333-43.

24. Subramanian S, Sridharan N, Balasundaram V, Chaudhari S. Efficacy and safety of Nimotuzumab in Unresectable, recurrent, and/or metastatic squamous cell carcinoma of the head and neck: a hospital-based retrospective evidence. South Asian J Cancer. 2018;7:188-92. 
25. Eriksen JG, Maare C, Johansen J, et al. Evaluation of the EGFR-inhibitor zalutumumab given with primary curative (chemo)radiation therapy to patients with squamous cell carcinoma of the head and neck: results of the DAHANCA 19 randomised phase 3 trial. Int J Radiat Oncol Biol Phys. 2014;88:465.

26. Mesía R, Henke M, Fortin A, et al. Chemoradiotherapy with or without panitumumab in patients with unresected, locally advanced squamous-cell carcinoma of the head and neck (CONCERT-1): a randomised, controlled, open-label phase 2 trial. Lancet Oncol. 2015;16:208-20.

27. Giralt J, Trigo J, Nuyts S, et al. Panitumumab plus radiotherapy versus chemoradiotherapy in patients with unresected, locally advanced squamous-cell carcinoma of the head and neck (CONCERT-2): a randomised, controlled, open-label phase 2 trial. Lancet Oncol. 2015;16:221-32.

28. Patil VM, Noronha V, Joshi A, et al. A randomized phase 3 trial comparing Nimotuzumab plus Cisplatin Chemoradiotherapy versus Cisplatin Chemoradiotherapy alone in locally advanced head and neck cancer. Cancer. 2019;125:3184-97.

29. Cohen EE, Rosen F, Stadler WM, et al. Phase II trial of ZD1839 in recurrent or metastatic squamous cell carcinoma of the head and neck. J Clin Oncol. 2003;21:1980-7.

30. Kirby AM, A'Hern RP, D'Ambrosio C, et al. Gefitinib (ZD1839, Iressa) as palliative treatment in recurrent or metastatic head and neck cancer. Br J Cancer. 2006;94:631-6.

31. Cohen EE, Kane MA, List MA, et al. Phase II trial of gefitinib $250 \mathrm{mg}$ daily in patients with recurrent and/or metastatic squamous cell carcinoma of the head and neck. Clin Cancer Res. 2005;11:8418-24.

32. Burris HA, Hurwitz HI, Dees EC, et al. Phase I safety, pharmacokinetics, and clinical activity study of lapatinib (GW572016), a reversible dual inhibitor of epidermal growth factor receptor tyrosine kinases, in heavily pretreated patients with metastatic carcinomas. J Clin Oncol. 2005;23:5305-13.

33. Del Campo JM, Hitt R, Sebastian P, et al. Effects of lapatinib monotherapy: results of a randomised phase II study in therapy-naive patients with locally advanced squamous cell carcinoma of the head and neck. Br J Cancer. 2011;105:618-27.

34. Seiwert TY, Fayette J, Cupissol D, et al. A randomized, phase II study of afatinib versus cetuximab in metastatic or recurrent squamous cell carcinoma of the head and neck. Ann Oncol. 2014;25:1813-20.

35. Stewart JS, Cohen EE, Licitra L, et al. Phase III study of gefitinib compared with intravenous methotrexate for recurrent squamous cell carcinoma of the head and neck. J Clin Oncol. 2009;27:1864-71.

36. Machiels JP, Haddad RI, Fayette J, et al. Afatinib versus methotrexate as second-line treatment in patients with recurrent or metastatic squamous-cell carcinoma of the head and neck progressing on or after platinum-based therapy (LUX-Head \& Neck 1): an open-label, randomised phase 3 trial. Lancet Oncol. 2015;16:583-94.

37. Harrington K, Berrier A, Robinson M, et al. Randomised phase II study of oral lapatinib combined with chemoradiotherapy in patients with advanced squamous cell carcinoma of the head and neck: rationale for future randomised trials in human papilloma virus-negative disease. Eur J Cancer. 2013;49:1609-18.

38. Harrington K, Temam S, Mehanna H, et al. Post-operative adjuvant lapatinib and concurrent chemoradiotherapy, followed by maintenance lapatinib monotherapy in high-risk patients with resected squamous cell carcinoma of the head and neck: a phase III, randomized, double-blind, placebo-controlled study. J Clin Oncol. 2015;33:4202-9.

39. Burtness B, Bourhis JP, Vermorken JB, et al. Afatinib versus placebo as adjuvant therapy after chemoradiation in a double-blind, phase III study (LUX-Head \& Neck 2) in patients with primary unresected, clinically intermediate-to-high-risk head and neck cancer: study protocol for a randomized controlled trial. Trials. 2014;15:469. 
40. Burtness B, Haddad R, Dinis J, et al. Afatinib vs placebo as adjuvant therapy after Chemoradiotherapy in squamous cell carcinoma of the head and neck: a randomized clinical trial. JAMA Oncol. 2019;5:1170-80.

41. Couzin-Frankel J. Cancer immunotherapy. Science. 2013;342:1432-3.

42. Joseph RW, Elassaiss-Schaap J, Kefford R, et al. Baseline tumor size is an independent prognostic factor for overall survival in patients with melanoma treated with Pembrolizumab. Clin Cancer Res. 2018;24:4960-7.

43. Eggermont AMM, Blank CU, Mandala M, et al. Adjuvant Pembrolizumab versus placebo in resected stage III melanoma. N Engl J Med. 2018;378:1789-801.

44. Weber J, Mandala M, Del Vecchio M, et al. Adjuvant Nivolumab versus Ipilimumab in resected stage III or IV melanoma. N Engl J Med. 2017;377:1824-35.

45. Antonia SJ, Villegas A, Daniel D, et al. Durvalumab after Chemoradiotherapy in stage III nonsmall-cell lung cancer. N Engl J Med. 2017;377:1919-29.

46. Antonia SJ, Villegas A, Daniel D, et al. Overall survival with Durvalumab after Chemoradiotherapy in stage III NSCLC. N Engl J Med. 2018;379:2342-50.

47. ht tps://www.pfizer.com/news/press-release/press-release-detail/ emd_serono_and_pfizer_provide_update_on_phase_iii_javelin_head_and_neck_100_study

48. https://clinicaltrials.gov/ct $2 /$ results? cond=KEYNOTE $+412 \&$ term $=\&$ cntry $=\&$ state $=\& c i$ $\mathrm{ty}=\&$ dist $=$.

49. https://clinicaltrials.gov/ct2/show/NCT03452137

Open Access This chapter is licensed under the terms of the Creative Commons Attribution 4.0 International License (http://creativecommons.org/licenses/by/4.0/), which permits use, sharing, adaptation, distribution and reproduction in any medium or format, as long as you give appropriate credit to the original author(s) and the source, provide a link to the Creative Commons license and indicate if changes were made.

The images or other third party material in this chapter are included in the chapter's Creative Commons license, unless indicated otherwise in a credit line to the material. If material is not included in the chapter's Creative Commons license and your intended use is not permitted by statutory regulation or exceeds the permitted use, you will need to obtain permission directly from the copyright holder. 